\title{
Genetic relatedness and risk factor analysis of ampicillin-resistant and high-level gentamicin- resistant enterococci causing bloodstream infections in Tanzanian children
}

Håvard Aamodt ${ }^{1,2}$, Stein Christian Mohn ${ }^{2}$, Samuel Maselle ${ }^{3}$, Karim P Manji ${ }^{4}$, Rob Willems ${ }^{5}$, Roland Jureen ${ }^{6}$, Nina Langeland ${ }^{1,2}$ and Bjørn Blomberg ${ }^{1,2^{*}}$

\begin{abstract}
Background: While enterococci resistant to multiple antimicrobials are spreading in hospitals worldwide, causing urinary tract, wound and bloodstream infections, there is little published data on these infections from Africa.

Methods: We assessed the prevalence, susceptibility patterns, clinical outcome and genetic relatedness of enterococcal isolates causing bloodstream infections in children in a tertiary hospital in Tanzania, as part of a prospective cohort study of bloodstream infections among 1828 febrile children admitted consecutively from August 2001 to August 2002.

Results: Enterococcal bacteraemia was identified in 2.1\% (39/1828) of admissions, and in 15.3\% (39/255) of cases of culture-confirmed bloodstream infections. The case-fatality rate in children with Enterococcus faecalis septicaemia $(28.6 \%, 4 / 14)$ was not significantly different from those with Enterococcus faecium septicaemia $(6.7 \%, 1 / 15, p=0.12)$. E. faecium isolates commonly had combined ampicillin-resistance and high-level gentamicin resistance (HLGR), (9/17), while E. faecalis frequently displayed HLGR (6/15), but were ampicillin susceptible. None of the tested enterococcal isolates displayed vancomycin resistance by Etest or PCR for vanA and vanB genes. Multi-locus sequence-typing (MLST) showed that the majority of E. faecium (7/12) belonged to the hospital associated Bayesian Analysis of Population Structure (BAPS) group 3-3. Pulsed-field gel electrophoresis (PFGE) indicated close genetic relationship particularly among E. faecium isolates, but also among $E$. faecalis isolates. There was also correlation between BAPS group and PFGE results. Risk factors for enterococcal bloodstream infection in univariate analysis were hospital-acquired infection and clinical diagnosis of sepsis with unknown focus. In multivariate analysis, neonates in general were relatively protected from enterococcal infection, while both prematurity and clinical sepsis were risk factors. Malnutrition was a risk factor for enterococcal bloodstream infection among HIV negative children.

Conclusion: This is the first study to describe bloodstream infections caused by ampicillin-resistant HLGR E. faecium and HLGR E. faecalis in Tanzania. The isolates of E. faecium and E. faecalis, respectively, showed high degrees of relatedness by genotyping using PFGE. The commonly used treatment regimens at the hospital are insufficient for infections caused by these microbes. The study results call for increased access to microbiological diagnostics to guide rational antibiotic use in Tanzania.
\end{abstract}

Keywords: Enterococcus, Enterococcus faecalis, Enterococcus faecium, Microbial drug resistance, Sepsis, Bacteremia, Pulsed-field gel electrophoresis, Multilocus sequence typing, Tanzania, Africa

\footnotetext{
* Correspondence: bjorn.blomberg@k2.uib.no

'Department of Clinical Science, University of Bergen, Bergen, Norway

${ }^{2}$ Center for Tropical Infectious Diseases, Department of Medicine, Haukeland

University Hospital, Bergen, Norway

Full list of author information is available at the end of the article
} 


\section{Background}

Enterococci are normal inhabitants of human and animal intestine [1-3]. They were previously considered innocent commensal organisms as compared to more virulent organisms, such as Streptococcus pyogenes and Staphylococcus aureus. In recent years enterococci, first Enterococcus faecalis [4] and more recently Enterococcus faecium [5,6], have emerged as major pathogens causing nosocomial infections, particularly urinary tract, wound and bloodstream infections. Enterococci tend to cause infections in immunocompromised hosts and seriously ill patients and patients with indwelling devices, such as catheters [7]. There is evidence that enterococci are a significant cause of bacteraemia in people with HIV infection [8]. Enterococci are intrinsically resistant to cephalosporins [9-11] and have reduced susceptibility to aminoglycosides [12]. Enterococci also have great ability to acquire antimicrobial resistance through mutations and exchange of genetic material [3]. High consumption of cephalosporins tends to select for enterococci and favour their spread in hospital settings [13-15]. Resistance to anti-enterococcal first-line drugs such as ampicillin and the combination ampicillin/gentamicin, commonly used empirically for sepsis, is increasing rapidly [16,17]. Vancomycin-resistant enterococci (VRE) have spread globally [16] and have become a very important cause of nosocomial infections. Treatment of VRE infections is hampered by the fact that many isolates are resistant to multiple other agents as well $[18,19]$. Typing of E. faecium strains by multi-locus sequence typing (MLST) has identified hospital-adapted subpopulations, initially coined clonal complex 17 (CC17), as being associated with serious infections and nosocomial outbreaks worldwide [16,20-22]. Further analysis on the population genetics of E. faecium indicated the existence of three hospital lineages (17, 18, and 78), that group in two Bayesian Analysis of Population Structure (BAPS) groups, BAPS 2-1 (lineage 78) and BAPS 3-3 (lineage 17 and 18) [23]. Recent whole genome-based phylogeny suggests that these hospitallineages are all part of a single clade, called A1 [24].

Little is known about the role of enterococci in bloodstream infections in East Africa, their resistance patterns, and whether certain clonal complexes are associated with more disease in this setting. However, carriage of highlevel gentamicin-resistant (HLGR) enterococci has been described in Zimbabwe [25] and South Africa [26]. Outbreaks of infections caused by VRE have been reported from South Africa [27] and VRE are often found in milk, beef, and chicken in Botswana [28].

The purpose of this study was to assess the prevalence, resistance patterns, and genetic relatedness of enterococcal isolates causing bloodstream infections in children at Muhimbili National Hospital, Dar es Salaam, Tanzania, and risk factors for such infections.

\section{Methods}

The study took place from August 2001 to August 2002 at the Paediatric Department at Muhimbili National Hospital, a tertiary referral hospital in Dar es Salaam, Tanzania. A cohort of 1828 consecutive admissions of a total of 1787 children presenting with clinical features of systemic infection were included in the study as described elsewhere [29]. The inclusion criteria were fever, hypothermia and other signs and symptoms described in the World Health Organization's program for Integrated Management of Childhood Illness (IMCI) [30], including general danger signs such as convulsions, lethargy, inability to drink or breastfeed, vomiting, and other signs of infection, such as neck stiffness, bulging fontanelles, cough, tachypnoea, difficulty breathing, chest in-drawings, nasal flaring, grunting, diarrhoea, dehydration, ear or eye discharge, lymphadenopathy, and, in neonates, signs of infection in the skin and umbilicus. The attending clinician recorded patient data using a standardized case report form. Medical records and departmental registries for admissions, discharges and deaths were reviewed with the purpose of quality-assuring the data.

The protocol was approved by The Research and Publications Committee of Muhimbili University College of Health Sciences and by the National AIDS Control Programme. Since the study subjects were young children aged 0-7 years, the parents or other accompanying, responsible family members were asked for written consent on behalf of the patient. Written informed consent was obtained before including the patient in the study and before taking blood tests. In some circumstances, in the case of critically ill patients, blood specimens were taken based on verbal consent, since these investigations are strongly recommended as routine investigations for severely ill febrile children, and since it would be inappropriate to delay management of such patients due to paperwork. The responsible family member was then approached in retrospect for written consent to use these specimens. The Tanzanian national language, Kiswahili, was used for obtaining consent.

Blood specimens were examined for growth of bacteria, mycobacteria and fungi by the use of BACTEC ${ }^{\mathrm{m}}$ $\mathrm{MYCO} / \mathrm{F}$ Lytic culture vials. Isolates were identified by standard microbiological methods as described in Mackie \& McCartney Practical Medical Microbiology [31]. E. faecalis and E. faecium species identification was confirmed by polymerase chain reaction (PCR) of the $d d l$ gene [32]. Susceptibility was tested by the disk diffusion method according to the guidelines of the National Committee for Clinical Laboratory Standards (NCCLS, now CLSI - Clinical and Laboratory Standards Institute) [33] for doxycycline and chloramphenicol. Testing for minimum inhibitory concentration (MIC) was performed by Etest (BioMerieux, Craponne, France, previously AB Biodisk, Solna, Sweden) 
for ampicillin, high-level gentamicin-resistance, vancomycin, linezolid, and quinupristin-dalfopristin. Resistance to ampicillin was defined as $\mathrm{MIC} \geq 16(\mathrm{mcg} / \mathrm{ml})$ and highlevel gentamicin-resistance as $\mathrm{MIC}>500(\mathrm{mcg} / \mathrm{ml})$ as assessed by Etest. PCR for vanA and $v a n B$ genes was performed to detect vancomycin resistance.

Genetic relatedness of the isolates was assessed by pulsed field gel electrophoresis (PFGE). This was performed on SmaI (Promega Corp, Madison, WI) digested genomic DNA as previously described [34] and modified by Dahl et al. [35]. The PFGE patterns were analysed with Bionumerics, version 3.0 (Applied Maths, Kortrijk, Belgium). The Dice coefficient of similarity was calculated, and unweighted pair group method with arithmetic averages (UPMGA) was used for cluster analysis. Twelve $E$. faecium isolates were also investigated by MLST. MLST was carried out with a standard set of primers that amplify the 7 genes included in the E. faecium MLST scheme [36]. Information on these loci, the latest set of primers, amplification conditions, and details of all isolates are available on the MLST Web site (http://pubmlst.org/efaecium/).

Community-acquired (CA) infection was defined as bloodstream infection (BSI) with growth of pathogenic bacteria in a blood-culture obtained within the first 48 hours after admission [37]. A neonate who was born in hospital within the last 10 days was considered as having hospital-acquired (HA) infection. HIV testing was performed by ELISA as previously described and confirmed by PCR for children $<18$ months [29]. For the univariate analysis, Chi-square test and student $t$-test was used to compare proportions and means, respectively. Multivariate analysis was performed by stepwise backwards logistic regression with threshold $\mathrm{p}=0.2$ for removal from the model and $p=0.05$ for inclusion. Risk factors were removed one-by-one starting with the least significant factor until remaining with only factors significant to the level of $\mathrm{p}<0.2$. Since only about half $(\mathrm{n}=942)$ of the study participants consented to be HIV tested, and the many missing values would affect the logistic regression model, we present two different models; one where HIV status is removed from the model $(\mathrm{n}=1603)$, and one where HIV status is locked in the model $(n=842)$. Statistical analysis was performed in Stata 13 (Stata Corp, College Station, Texas, US).

\section{Results}

Thirty-nine of 1828 cases (2.1\%) had growth of enterococcal isolates in blood culture. The clinical presentation of patients with enterococcal bacteraemia was assessed as clinical sepsis without certain focus in $45 \%(n=15)$, pneumonia in $21 \%(\mathrm{n}=7)$, urinary tract infection in $11 \%$ $(\mathrm{n}=4)$, and non-specific infection without sepsis-symptoms in $21 \%(n=7)$, while six patients could not be evaluated due to missing clinical information. Enterococcal isolates contributed to $15.3 \%$ (39/255) of the total number of identified episodes of bacteraemia in the study, 17 caused by E. faecium, 15 by $E$. faecalis and 7 by enterococcal isolates that were not available for speciation. Since some patients had more than one isolate of $E$. faecium, the total number of E. faecium isolates was 21. Twenty-three percent (9/39) of the patients had enterococci as part of a polymicrobial infection, involving other enterococcal isolates as well as 2 isolates of Candida species, 3 klebsiellae isolates and single isolates of Salmonella enteritidis, Staphylococcus aureus and Streptococcus viridans. The majority of E. faecium bacteraemia episodes (10/17) were hospital-acquired, i.e. obtained in blood-cultures taken $\geq 48$ hours after admission, while the majority of E. faecalis bacteraemia episodes (10/15) were community-acquired.

The antimicrobial susceptibility of the isolates is shown in Table 1 . Nine of 17 E. faecium isolates showed combined ampicillin-resistance and HLGR. Six of 15 E. faecalis isolates were HLGR, but none of these were resistant to ampicillin. All except one of the HLGR isolates were also resistant to chloramphenicol. The resistant strains were recovered from different wards, including the neonatal ward. All isolates were sensitive to vancomycin by disk-diffusion testing and Etest, and none of the 22 tested isolates were positive on PCR for van $A$ or $v a n B$ genes.

Twenty-one isolates of E. faecium and 15 isolates of E. faecalis were available for PFGE. The majority of the E. faecium and E. faecalis isolates were closely related as assessed visually from PFGE (Figure 1). From the figure $11 / 15$ E. faecalis cluster at $72 \%$ similarity and $13 / 21$ E. faecium at $80 \%$ similarity (Some duplicate strains are analysed in the dendrogram, resulting in totals of $12 / 16$ similarly clustering $E$. faecalis and $17 / 25$ similarly clustering $E$. faecium banding patterns). To assess whether the E. faecium isolates belonged to previously described, globally dispersed hospital subpopulations, MLST was performed on 12 E. faecium isolates and identified 7 different sequence types (STs) of which ST132 (5 isolates) and ST18 (2 isolates) were found in multiple isolate. These two STs, involving 7/12 isolates belong to lineage18 , which is contained in the global hospital-associated BAPS group 3-3. In the current study, persons with hospital-acquired infection accounted for 4 of 6 isolates in BAPS group 3-3. All 5 isolates of ST132 came from the same ward (neonatal ward). Isolates with ST125 and ST169, belonging to BAPS 3-1, and ST170 and ST171 belonging to BAPS 2-3 are also evolutionary related. Isolate 1064, ST178, belongs to BAPS 1 , which is significantly associated with human commensal isolates in the community. Isolates with STs belonging to BAPS 3-3 were also related by PFGE. All isolates with STs belonging to BAPS 3-3 as well as isolate 2118 (ST125) belonging to BAPS 3-1 were ampicillin resistant, while the 


\begin{tabular}{|c|c|c|c|c|c|c|c|}
\hline \multirow{3}{*}{$\begin{array}{l}\text { Antibiotic } \\
\text { Antibiotic }\end{array}$} & \multicolumn{7}{|c|}{ Susceptibility } \\
\hline & \multicolumn{3}{|c|}{ E. faecium } & \multicolumn{3}{|c|}{ E. faecalis } & \multirow{2}{*}{$\begin{array}{l}\text { Enterococc } \\
\text { All }\end{array}$} \\
\hline & CA & HA & All & CA & $\mathrm{HA}$ & All & \\
\hline \multicolumn{8}{|l|}{ Etest } \\
\hline Ampicillin & $25 \%$ & $11 \%$ & $19 \%$ & $100 \%$ & $100 \%$ & $100 \%$ & $53 \%$ \\
\hline High-level Gentamicin & $67 \%$ & $33 \%$ & $52 \%$ & $56 \%$ & $67 \%$ & $60 \%$ & $56 \%$ \\
\hline Vancomycin* & $100 \%$ & $100 \%$ & $100 \%$ & $100 \%$ & $100 \%$ & $100 \%$ & $100 \%$ \\
\hline Linezolid & $100 \%$ & $100 \%$ & $100 \%$ & $100 \%$ & $100 \%$ & $100 \%$ & $100 \%$ \\
\hline Quinupristin-dalfopristin & $100 \%$ & $100 \%$ & $100 \%$ & $0 \%$ & $17 \%$ & $7 \%$ & $61 \%$ \\
\hline \multicolumn{8}{|l|}{ Disk-diffusion } \\
\hline Chloramphenicol & $50 \%$ & $11 \%$ & $32 \%$ & $38 \%$ & $33 \%$ & $36 \%$ & $33 \%$ \\
\hline Doxycycline & $9 \%$ & $0 \%$ & $5 \%$ & $25 \%$ & $0 \%$ & $14 \%$ & $9 \%$ \\
\hline
\end{tabular}

*Confirmed by negative PCR for vanA and vanB genes.

$\mathrm{CA}=$ community-acquired, $\mathrm{HA}=$ hospital-acquired.

other isolates were ampicillin susceptible. None of the isolates carried the esp gene.

Time in hospital from admission until blood cultures were obtained was not significantly longer among patients with BSI caused by E. faecium (median 3 days, range $0-10, \mathrm{p}=0.06$ ) or $E$. faecalis (median 4 days, range $0-20, \mathrm{p}=0.56)$ compared to those with BSI caused by other microbes (median 2 days, range $0-34$ ).

Table 2 shows univariate and multivariate analysis of risk factors for enterococcal BSI. HIV status was only known for $51.5 \%(\mathrm{n}=942)$ of patients, among which $18.7 \%(\mathrm{n}=176)$ were positive. As including HIV status in the multivariate analysis would eliminate close to half of the observations, we present two different multivariate models; one without HIV status ( $n=1603$ evaluable patients) and one with HIV status locked in the model $(\mathrm{n}=842)$. Apart from factors shown in the table, all recorded clinical diagnoses and symptoms were assessed as risk factors.

Hospital-acquired infection (i.e. positive blood-culture drawn $>48$ hours after admission) was a risk factor for enterococcal BSI compared to community-acquired clinical infections (i.e. positive blood-culture drawn $<48$ hours after admission) in univariate analysis (Odds ratio (OR): 1.9, 95\% confidence interval $(95 \% \mathrm{CI}): 1.01-3.61, \mathrm{p}=$ $0.046)$, but did not remain a significant risk factor in multivariate analysis (OR: 2.0, 95\% CI: 0.96-4.00, p = 0.066) (Table 2). There was no difference in proportions of hospital-acquired infection between enterococcal BSI compared to patients with other culture-proven BSI (44\%, 17/ 39 vs $51 \%, 111 / 216, p=0.37)$. Community-acquired and hospital-acquired E. faecium isolates did not have different rates of ampicillin-resistance $(75 \% 9 / 12$ vs $89 \%, 8 / 9, \mathrm{p}=$ $0.42)$ or high-level gentamicin resistance $(38 \%, 8 / 21$ vs $53 \%$, $8 / 15, \mathrm{p}=0.36)$.
A clinical diagnosis of sepsis with unspecified focus of infection was a borderline significant risk factor for enterococcal infection in univariate analysis (OR: 2.0, 95\% CI: $1.00-4.00, \mathrm{p}=0.050)$ and a significant risk factor in multivariate analysis (OR: $2.9,95 \%$ CI: 1.36-6.20, $\mathrm{p}=0.006)$. In the multivariate analysis on the subset with known HIV status, sepsis remained a significant risk factor. Clinical diagnoses of urinary tract infection or pneumonia were not significant risk factors.

Prematurity was a significant risk factor for enterococcal bacteraemia in multivariate analysis (OR: 4.4, 95\% CI: 1.06-18.1, $\mathrm{p}=0.041$ ), although this relationship was not significant in univariate analysis. Being a neonate appeared relatively protective in multivariate analysis (OR: 0.4, 95\% CI: 0.14-0.98, $\mathrm{p}=0.046$ ), although again this was not significant in the univariate analysis. Within the cohort of neonates (children $<1$ month of age), enterococcal BSI accounted for almost a quarter of BSI episodes in premature babies $(23.1 \%, 3 / 13)$, and, despite the small numbers, the premature babies had a significantly increased risk of enterococcal BSI compared to babies born to term $(5.8 \%$ vs $1.4 \%$, OR 4.3, 95\% CI: $1.03-17.55, \mathrm{p}=0.045)$. Prematurity remained a significant risk factor in the multivariate analysis on the subset of children with known HIV status.

The proportion of malnourished children was $21 \%$ in children with enterococcal BSI and 15\% in controls, but this difference was not significant, neither in the univariate nor in the overall multivariate analysis. However, in the multivariate analysis on patients with known HIV status, malnutrition was indeed a significant risk factor for enterococcal BSI (OR 3.7, 95\% CI 1.24-11.28, $\mathrm{p}=$ 0.019). The selection of children with known HIV status may have been skewed in the sense that malnourished and HIV positive patients may share clinical features, and thus more malnourished children may have been 


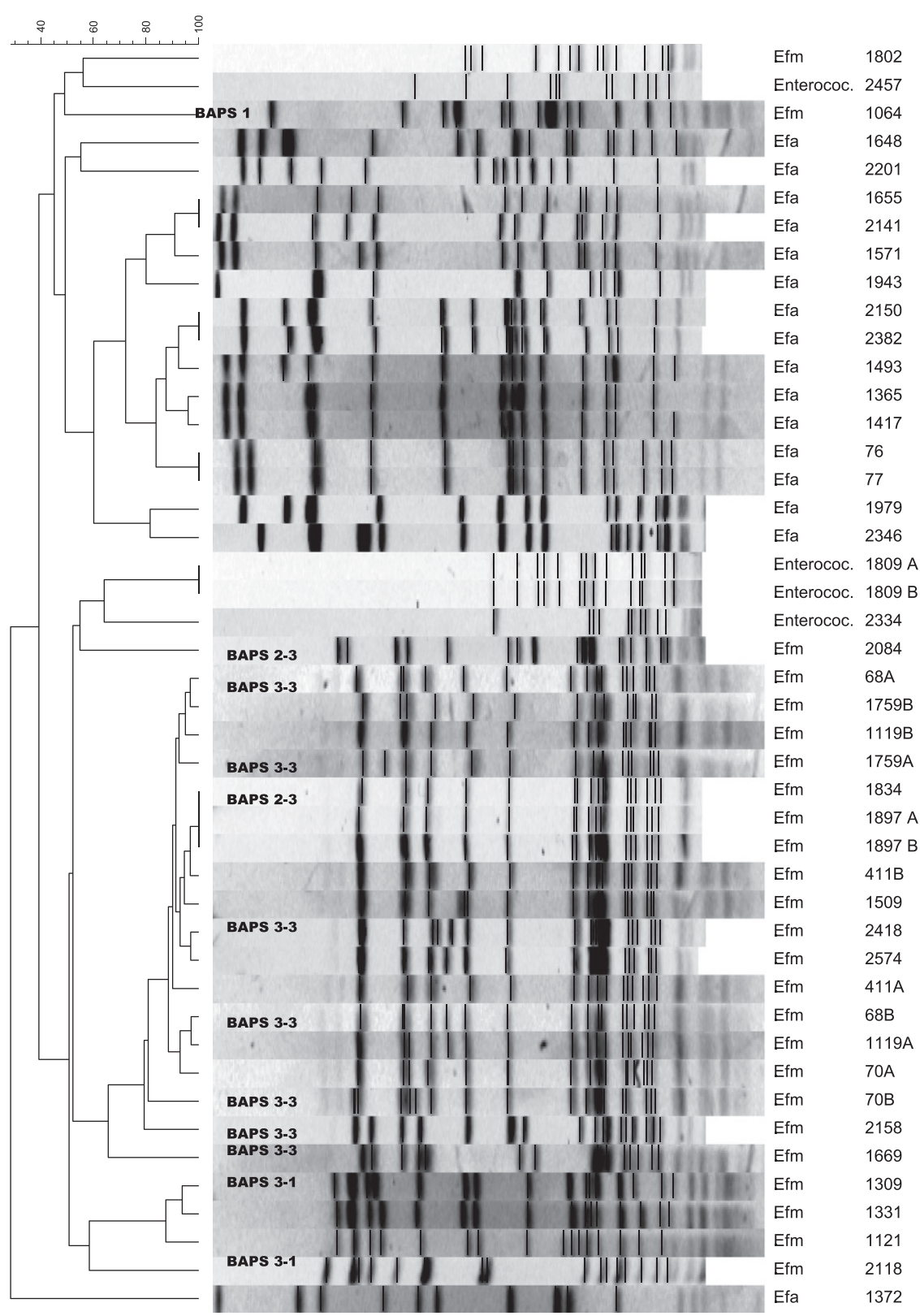

Figure 1 Genetic relatedness of enterococcal isolates causing bloodstream infections in Tanzanian children. The dendrogram shows genetic relatedness based on pulsed-field gel electrophoresis analysis on Smal digested genomic DNA. Genetic relatedness was also assessed by multi-locus sequence typing and corresponding Bayesian Analysis of Population Structure (BAPS) groups are indicated in the dendrogram.

tested for HIV on clinical suspicion. Indeed, within the cohort of HIV negative children, malnourishment was a statistically significant risk factor for enterococcal BSI (6.6\% (5/76) vs 2,3\% (14/606), OR: 3.0, 95\% CI: $1.04-$ $8.51, \mathrm{p}=0.042)$, while there was no such association in HIV positive children $(1.7 \%(1 / 60)$ vs $2.0 \%(2 / 100)$, OR: 0.83, 95\% CI: 0.07-9.36, $\mathrm{p}=0.881)$.

Altogether, HIV status was known for 51.5\% (942/ 1828) of the total number of admission during the study (corresponding to $51.0 \%$ of patients $(911 / 1787)$, and for $59 \%(23 / 39)$ of those with enterococcal BSI. There was no significant difference in HIV positivity rate between patients with enterococcal BSI $(13.0 \%, 3 / 23)$ and all other patients with known HIV status (18.8\%, 173/919, $\mathrm{p}=0.486$ ), and neither compared to those with BSI of other aetiologies $(22.0 \%, 28 / 127, \mathrm{p}=0.33)$. Neither cephalosporin use, nor other antibiotic use prior to obtaining blood-culture, were significant risk factors for enterococcal 
Table 2 Univariate and multivariate† analysis of risk factors for enterococcal bloodstream infection among febrile children admitted to Muhimbili National Hospital $(n=1828)^{*}$

\begin{tabular}{|c|c|c|c|c|c|}
\hline \multirow[b]{3}{*}{ Variable } & \multicolumn{3}{|c|}{ Univariate anlysis } & \multicolumn{2}{|l|}{ Multivariate analyses $^{\dagger}$} \\
\hline & \multirow[b]{2}{*}{ Enterococci } & \multirow[b]{2}{*}{ Others } & \multirow[b]{2}{*}{ OR $(95 \% \mathrm{Cl}) \mathrm{P}$} & \multirow{2}{*}{$\begin{array}{l}\text { All patients with clinical } \\
\text { information }(n=1603) \\
\text { OR }(95 \% \mathrm{Cl}) \mathrm{P}\end{array}$} & \multirow{2}{*}{$\begin{array}{l}\text { All patients with known } \\
\text { HIV status }(\mathrm{n}=842) \\
\text { OR }(95 \% \mathrm{CI}) \mathrm{P}\end{array}$} \\
\hline & & & & & \\
\hline Neonate & $28.2 \%(11 / 39)$ & $29.3(524 / 1789)$ & $0.9(0.47-1.92) 0.883$ & $0.4(0.14-0.98) 0.046$ & ${ }^{\ddagger} g$ \\
\hline Prematurity & $9.1(3 / 33)$ & $3.2(51 / 1570)$ & $3.0(0.88-10.08) 0.079$ & $4.4(1.06-18.1) 0.041$ & $4.7(1.19-18.8) 0.019$ \\
\hline Male sex & $61.5(24 / 39)$ & $55.4(992 / 1789)$ & $1.3(0.67-2.47) 0.450$ & ${ }^{\ddagger} \mathrm{e}$ & $2.9(1.03-7.91) 0.043$ \\
\hline Hospital acquired infection ${ }^{\#}$ & $48.7(19 / 39)$ & $33.2(594 / 1789)$ & $1.9(1.01-3.61) 0.046$ & $2.0(0.96-4.00) 0.066$ & $1.8(0.73-4.29) 0.209$ \\
\hline Antibiotic use before blood culture & $66.7(26 / 39)$ & $57.0(1020 / 1789)$ & $1.5(0.77-2.95) 0.231$ & ${ }^{\ddagger} \mathrm{C}$ & ${ }^{*} \mathrm{~b}$ \\
\hline Cephalosporin use before blood culture & $5.1(2 / 39)$ & $4.0 \%(71 / 1789)$ & $1.3(0.31-5.53) 0.715$ & ${ }^{\ddagger} \mathrm{a}$ & ${ }^{\ddagger} \mathrm{C}$ \\
\hline \multicolumn{6}{|l|}{ Diagnoses } \\
\hline Malnutrition & $21.2(7 / 33)$ & $15.4(241 / 1570)$ & $1.5(0.64-3.46) 0.360$ & $\ddagger_{f}$ & $3.7(1.24-11.28) 0.019$ \\
\hline HIV infection & $13.0(3 / 23)$ & $18.8(173 / 919)$ & $0.6(0.19-2.20) 0.486$ & $\S$ & $0.5(0.14-2.04) 0.363^{\S}$ \\
\hline Urinary tract infection & $12.1 \%(4 / 33)$ & $11.4 \%(179 / 1570)$ & $1.1(0.37-3.08) 0.898$ & ${ }^{\ddagger} \mathrm{b}$ & ${ }^{\ddagger} \mathrm{a}$ \\
\hline Pneumonia & $21.2 \%(7 / 33)$ & $20.1 \%(315 / 1570)$ & $1.1(0.46-2.49) 0.871$ & ${ }^{\ddagger} \mathrm{d}$ & ${ }^{\ddagger} e$ \\
\hline Sepsis with unknown focus & $45.5 \%(15 / 33)$ & $29.4 \%(462 / 1570)$ & $2.0(1.00-4.00) 0.050$ & $2.9(1.36-6.20) 0.006$ & $2.6(1.06-6.55) 0.037$ \\
\hline Congenital heart disease & $6.1 \%(2 / 33)$ & $2.3 \%(36 / 1570)$ & $2.7(0.63-11.93) 0.177$ & ${ }^{\ddagger} g$ & ${ }^{\ddagger} \mathrm{d}$ \\
\hline Acute watery diarrhoea & $9.1(3 / 33)$ & $21.8(343 / 1570)$ & $0.4(0.11-1.18) 0.091$ & $0.3(0.09-1.07) 0.065$ & $f_{f}$ \\
\hline
\end{tabular}


BSI. Male sex was associated with enterococcal BSI in the multivariate analysis of the subset with known HIV status, but not in univariate or overall multivariate analysis (Table 2).

Clinical outcome was known for 89.3\% (1632/1828) of the admissions. Among those with known outcome, the case-fatality rate in children with $E$. faecalis septicaemia $(28.6 \%, 4 / 14)$ was not significantly higher than for those with $E$. faecium septicaemia $(6.7 \%, 1 / 15, \mathrm{p}=0.12)$. Children with enterococcal BSI received inappropriate chemotherapy due to antimicrobial resistance in $44.1 \%$ $(15 / 39)$ of the cases, but this was not significantly different from BSI due to other agents $(41.6 \%, 77 / 185, \mathrm{p}=$ $0.8)$. The case fatality rate was $20 \%(3 / 15)$ for enterococcal BSI treated with inappropriate antibiotics, and $10 \%$ $(2 / 19)$ for those treated with appropriate antibiotics, however, the numbers were small and the difference was not statistically significant $(\mathrm{p}=0.439)$. Among patients with $E$. faecalis bacteraemia, two had concomitant malaria parasitaemia detected on microscopy, both of whom died. Among patients with E. faecium bacteraemia, one had concomitant growth of $S$. aureus, two had Candida species in blood culture, and two had concomitant malaria parasitaemia, none of whom died.

\section{Discussion}

Recent studies from Tanzania have described enterococci in urinary tract infections [38,39], but, to our knowledge, there are no published clinical studies on enterococcal bloodstream infections from this country. Although the data from the current study were collected some years ago, the study provides insight in prevalence, resistance patterns, and risk factors of enterococcal bloodstream infections in Tanzania, confirming similar trends as seen in Europe and the USA, and sets a benchmark for future studies. Surprisingly, we do not find that prior use of cephalosporins or other antibiotic use is a risk factor for enterococcal BSI in this study. This may be due to small numbers.

Enterococci are a prevalent aetiology for bloodstream infections in Tanzania, comprising $15.3 \%$ of the total number of identified episodes of bacteraemia in the current study. Information on antimicrobial use was available for $85.2 \%(1557 / 1828)$ of admissions and at least two-thirds $(67.2 \%, 1046 / 1557)$ received antibiotics before bloodculture was taken. This may have selected more resistant clones in our study. All ampicillin-resistant HLGR E. faecium isolates were susceptible to vancomycin, linezolid and quinupristin/dalfopristin. All HLGR E. faecalis isolates were sensitive to vancomycin, linezolid and ampicillin.

As expected, the majority of E. faecium isolates were hospital-acquired, and hospital-acquired infection was a significant risk factor for enterococcal infections in univariate analysis. In accordance with other studies, most isolates associated with hospital-acquired infections clustered in BAPS group 3-3 based on MLST. The patient with ST178/ BAPS 1, a BAPS group significantly associated with human commensals in the community, had a clinical diagnosis of tuberculosis/pneumonia and anaemia.

A clinical diagnosis of sepsis, but not pneumonia and urinary tract infection, was a significant risk factor for enterococcal BSI. While neonates in general were not particularly at risk, premature babies had significantly increased risk of enterococcal BSI. Malnutrition was a significant risk factor for enterococcal BSI in subanalysis of children with known HIV status (multivariate analysis), particularly among those with known negative HIV test (univariate analysis).

Among the 14 patients with $E$. faecium recovered from blood culture (and known outcome) only one died, this was a two months old boy with suspected meningitis who was admitted with fever, convulsions and neck stiffness. The isolate was resistant to ampicillin and chloramphenicol, and high-level gentamicin-resistant. The patient received antimicrobial treatment with penicillin and chloramphenicol, to which the enterococcal isolate was resistant. Chloramphenicol is largely abandoned in developed countries due to the risk of aplastic anaemia, although it has been used to treat VRE. Chloramphenicol is still widely used in Africa for the treatment of a number of bacterial infections including meningitis and typhoid fever. It was, however, uncertain whether the E. faecium isolate was the cause of the meningitis, which was thought to be the main diagnosis.

Among the patients with E. faecalis bacteraemia, four died. Two of those who died had concomitant malaria parasitaemia on microscopy, one of which received quinine (for the other therapy was unknown). Two of them had HLGR, including one of the patients with malaria parasitaemia. The given antimicrobial therapy was known for three of the patients, one of which received inappropriate antibiotic treatment (chloramphenicol), and two of which received at least one appropriate antibiotic (ampicillin) and one inappropriate (gentamicin) for HLGR.

\section{Conclusions}

Consistent with global trends showing increasing importance of E. faecium as a cause of human infections relative to E. faecalis [22,40], our study found that more than half of the enterococcal bloodstream infections were caused by E. faecium in this hospital. This first clinical study of enterococcal bloodstream infections from Tanzania has identified septicaemia caused by ampicillin-resistant and HLGR enterococci as common nosocomial infections with a considerable case-fatality rate. Premature babies and malnourished children, and those with a clinical diagnosis of sepsis with unknown focus appeared at particular risk. These infections 
constitute a therapeutic problem as the commonly used treatment regimens at the hospital (ampicillin and gentamicin or penicillin and chloramphenicol) are insufficient for infections caused by ampicillin-resistant HLGR enterococci. Vancomycin is the empirical drug of choice for proven E. faecium bloodstream infections. However, in a resource-constrained setting such as Tanzania, limited access to microbiological laboratories and expensive second-line antimicrobials may render such recommendations futile. Furthermore, escalating empirical use of broad-spectrum antibiotics may be ecologically detrimental in a setting where microbiological laboratory services are scarce and patients rarely get a bacteriologically confirmed diagnosis. In this context, it is crucial to counteract further emergent antibiotic resistance by infection control measures and prudent antibiotic use based on best available data from surveillance activities, sentinel surveys, and, whenever possible, microbiological diagnosis for the individual patient.

\section{Abbreviations \\ 95\% Cl: 95\% confidence interval; AIDS: Acquired immune deficiency syndrome; BAPS: Bayesian Analysis of Population Structure; BSI: Bloodstream infection; CA: Community-acquired; CC17: Clonal complex 17; CLSI: Clinical and Laboratory Standards Institute; ELISA: Enzyme-linked immunosorbent assay; HA: Hospital-acquired; HIV: Human immunodeficiency virus: HLGR: High-level gentamicin-resistance; IMCI: Integrated management of childhood illnesses; MIC: Minimum inhibitory concentration; MLST: Multi- locus sequence-typing; NCCLS: National Committee for Clinical Laboratory Standards; OR: Odds ratio; PCR: Polymerase chain reaction; PFGE: Pulsed-field gel electrophoresis; ST: Sequence type; UPMGA: Unweighted pair group method with arithmetic averages; VRE: Vancomycin-resistant enterococci.}

\section{Competing interests}

The authors declare that they have no competing interests.

\section{Authors' contributions}

$\mathrm{BB}, \mathrm{NL}, \mathrm{KPM}$ and SYM conceived and designed the study. BB, KPM and SYM supervised the data collection. BB, SCM, RJ and RW performed phenotypic and molecular microbiological analysis. RW performed multi-locus sequence-typing. $\mathrm{HA}$ and $\mathrm{BB}$ analysed the data. All authors contributed to the interpretation of the data and writing of the manuscript. All authors read and approved the final manuscript.

\section{Acknowledgements}

The study received funding from grant 100675 from the Norwegian Research Council and from the Norwegian Council for Higher Education's Programme for Development Research and Education under project number 44003 PRO 42.2.91. We are grateful to Dr Kristine Mørch, Head of the National Centre for Infectious Tropical Diseases, for support during data-analysis and writing of the manuscript. We would like to thank all doctors, nurses, laboratory technicians and other staff at Muhimbili National Hospital who contributed to the study.

\footnotetext{
Author details

${ }^{1}$ Department of Clinical Science, University of Bergen, Bergen, Norway. ${ }^{2}$ Center for Tropical Infectious Diseases, Department of Medicine, Haukeland University Hospital, Bergen, Norway. ${ }^{3}$ Department of Microbiology and Immunology, Muhimbili University of Health and Allied Sciences, Dar es Salaam, Tanzania. ${ }^{4}$ Department of Pediatrics and Child Health, Muhimbili University of Health and Allied Sciences, Dar es Salaam, Tanzania. ${ }^{5}$ Department of Medical Microbiology, University Medical Center Utrecht, Utrecht, Netherlands. ${ }^{6}$ National University Health System, Singapore City, Singapore.
}

Received: 30 September 2014 Accepted: 19 February 2015

Published online: 28 February 2015

\section{References}

1. Sghir A, Gramet G, Suau A, Rochet V, Pochart P, Dore J. Quantification of bacterial groups within human fecal flora by oligonucleotide probe hybridization. Appl Environ Microbiol. 2000;66(5):2263-6.

2. Damborg P, Top J, Hendrickx AP, Dawson S, Willems RJ, Guardabassi L. Dogs are a reservoir of ampicillin-resistant Enterococcus faecium lineages associated with human infections. Appl Environ Microbiol. 2009;75(8):2360-5.

3. Witte W, Wirth R, Klare I. Enterococci. Chemotherapy. 1999;45(2):135-45.

4. Low DE, Keller N, Barth A, Jones RN. Clinical prevalence, antimicrobial susceptibility, and geographic resistance patterns of enterococci: results from the SENTRY Antimicrobial Surveillance Program, 1997-1999. Clin Infect Dis. 2001:32 Suppl 2:S133-45.

5. Treitman AN, Yarnold PR, Warren J, Noskin GA. Emerging incidence of Enterococcus faecium among hospital isolates (1993 to 2002). J Clin Microbiol. 2005;43(1):462-3.

6. Bonten MJ, Willems RJ. Vancomycin-resistant enterococcus-chronicle of a foretold problem]. Nederlands Tijdschrift Voor Geneeskunde. 2012;156(38):A5233.

7. Oppenheim BA. The changing pattern of infection in neutropenic patients. J Antimicrob Chemother. 1998:41 Suppl D:7-11.

8. Pedro-Botet ML, Modol JM, Valles X, Romeu J, Sopena N, Gimenez M, et al. Changes in bloodstream infections in HIV-positive patients in a university hospital in Spain (1995-1997). Int J Infect Dis. 2002;6(1):17-22.

9. Kristich CJ, Little JL, Hall CL, Hoff JS. Reciprocal regulation of cephalosporin resistance in Enterococcus faecalis. mBio. 2011;2(6):e00199-00111.

10. Kristich CJ, Little JL. Mutations in the beta subunit of RNA polymerase alter intrinsic cephalosporin resistance in Enterococci. Antimicrob Agents Chemother. 2012;56(4):2022-7.

11. Georgopapadakou NH, Liu FY. Binding of beta-lactam antibiotics to penicillin-binding proteins of Staphylococcus aureus and Streptococcus faecalis: relation to antibacterial activity. Antimicrob Agents Chemother. 1980;18(5):834-6.

12. Moellering Jr RC, Weinberg AN. Studies on antibiotic syngerism against enterococci. II. Effect of various antibiotics on the uptake of 14 C-labeled streptomycin by enterococci. J Clin Invest. 1971:50(12):2580-4.

13. Paterson DL. "Collateral damage" from cephalosporin or quinolone antibiotic therapy. Clin Infect Dis. 2004;38 Suppl 4:S341-5.

14. Pallares R, Pujol M, Pena C, Ariza J, Martin R, Gudiol F. Cephalosporins as risk factor for nosocomial Enterococcus faecalis bacteremia. A matched case-control study. Arch Intern Med. 1993;153(13):1581-6.

15. Dahms RA, Johnson EM, Statz CL, Lee JT, Dunn DL, Beilman GJ. Thirdgeneration cephalosporins and vancomycin as risk factors for postoperative vancomycin-resistant enterococcus infection. Arch Surg. 1998;133(12):1343-6.

16. Willems RJ, Top J, van Santen M, Robinson DA, Coque TM, Baquero F, et al, Global spread of vancomycin-resistant Enterococcus faecium from distinct nosocomial genetic complex. Emerg Infect Dis. 2005;11(6):821-8.

17. Cattoir V, Leclercq R. Twenty-five years of shared life with vancomycinresistant enterococci: is it time to divorce? J Antimicrob Chemother. 2013;68(4):731-42.

18. Huycke MM, Sahm DF, Gilmore MS. Multiple-drug resistant enterococci: the nature of the problem and an agenda for the future. Emerg Infect Dis. 1998;4(2):239-49.

19. McCracken M, Wong A, Mitchell R, Gravel D, Conly J, Embil J, et al, Molecular epidemiology of vancomycin-resistant enterococcal bacteraemia: results from the Canadian Nosocomial Infection Surveillance Program, 1999-2009. J Antimicrob Chemother. 2013;68(7):1505-9.

20. Hsieh YC, Lee WS, Ou TY, Hsueh PR. Clonal spread of CC17 vancomycinresistant Enterococcus faecium with multilocus sequence type 78 (ST78) and a novel ST444 in Taiwan. Eur J Clin Microbiol Infect Dis. 2010;29(1):25-30.

21. Laverde Gomez JA, van Schaik W, Freitas AR, Coque TM, Weaver KE, Francia MV, et al. A multiresistance megaplasmid pLG1 bearing a hylEfm genomic island in hospital Enterococcus faecium isolates. Int J Med Microbiol. 2011;301(2):165-75.

22. Top J, Willems R, Blok H, de Regt M, Jalink K, Troelstra A, et al. Ecological replacement of Enterococcus faecalis by multiresistant clonal complex 17 Enterococcus faecium. Clin Microbiol Infect. 2007:13(3):316-9.

23. Willems RJ, Top J, van Schaik W, Leavis $H$, Bonten $M$, Siren J, et al. Restricted gene flow among hospital subpopulations of Enterococcus faecium. mBio. 2012;3(4):e00151-00112. 
24. Lebreton F, van Schaik W, McGuire AM, Godfrey P, Griggs A, Mazumdar V, et al. Emergence of epidemic multidrug-resistant Enterococcus faecium from animal and commensal strains. mBio. 2013;4(4):e00534-13.

25. Patterson JE, Barry M, Gallant J, Mangine LS, Farrel P, Latif A. Epidemiology of high-level gentamicin resistant enterococcal isolates from Zimbabwe. Am J Trop Med Hyg. 1990;43(4):397-9.

26. Keddy KH, Klugman KP, Liebowitz LD. Incidence of high-level gentamicin resistance in enterococci at Johannesburg Hospital. S Afr Med J. 1996;86(10):1273-6.

27. McCarthy KM, Van Nierop W, Duse A, Von Gottberg A, Kassel M, Perovic O, et al. Control of an outbreak of vancomycin-resistant Enterococcus faecium in an oncology ward in South Africa: effective use of limited resources. J Hosp Infect. 2000;44(4):294-300.

28. Chingwaru W, Mpuchane SF, Gashe BA. Enterococcus faecalis and Enterococcus faecium isolates from milk, beef, and chicken and their antibiotic resistance. J Food Prot. 2003;66(6):931-6.

29. Blomberg B, Manji KP, Urassa WK, Tamim BS, Mwakagile DS, Jureen R, et al. Antimicrobial resistance predicts death in Tanzanian children with bloodstream infections: a prospective cohort study. BMC Infect Dis. 2007;7:43.

30. IMCI Integrated Management of Childhood IIIness. Model Chapter for Textbooks. Document no. WHO/FCH/CAH/01.01. Accessible from http://whqlibdoc.who.int/hq/2001/WHO_FCH_CAH_01.01.pdf. Geneva: World Health Organization; 2001.

31. Collee JG, Marmion BP, Irvine R, Fraser AG, Simmons A. Mackie \& McCartney Practical Medical Microbiology. 14th ed. New York: Churchill Livingstone; 1996

32. Angeletti S, Lorino G, Gherardi G, Battistoni F, De Cesaris M, Dicuonzo G. Routine molecular identification of enterococci by gene-specific PCR and 165 ribosomal DNA sequencing. J Clin Microbiol. 2001;39(2):794-7.

33. NCCLS. Performance standards for antimicrobial disk susceptibility tests. Approved standard M2-M7. Wayne, Pa, USA 1997 (7th edition).

34. Murray BE, Singh KV, Heath JD, Sharma BR, Weinstock GM. Comparison of genomic DNAs of different enterococcal isolates using restriction endonucleases with infrequent recognition sites. J Clin Microbiol. 1990;28(9):2059-63.

35. Dahl KH, Simonsen GS, Olsvik O, Sundsfjord A. Heterogeneity in the vanB gene cluster of genomically diverse clinical strains of vancomycin-resistant enterococci. Antimicrob Agents Chemother. 1999;43(5):1105-10.

36. Homan WL, Tribe D, Poznanski S, Li M, Hogg G, Spalburg E, et al. Multilocus sequence typing scheme for Enterococcus faecium. J Clin Microbiol. 2002;40(6):1963-71.

37. Siegman-Igra Y, Fourer B, Orni-Wasserlauf R, Golan Y, Noy A, Schwartz D, et al. Reappraisal of community-acquired bacteremia: a proposal of a new classification for the spectrum of acquisition of bacteremia. Clin Infect Dis. 2002;34(11):1431-9.

38. Masinde A, Gumodoka B, Kilonzo A, Mshana SE. Prevalence of urinary tract infection among pregnant women at Bugando Medical Centre, Mwanza, Tanzania. Tanzan J Health Res. 2009;11(3):154-9.

39. Moyo SJ, Aboud S, Kasubi M, Maselle SY. Bacterial isolates and drug susceptibility patterns of urinary tract infection among pregnant women at Muhimbili National Hospital in Tanzania. Tanzan J Health Res. 2010;12(4):236-40.

40. Iwen PC, Kelly DM, Linder J, Hinrichs SH, Dominguez EA, Rupp ME, et al. Change in prevalence and antibiotic resistance of Enterococcus species isolated from blood cultures over an 8-year period. Antimicrob Agents Chemother. 1997;41(2):494-5

\section{Submit your next manuscript to BioMed Central and take full advantage of:}

- Convenient online submission

- Thorough peer review

- No space constraints or color figure charges

- Immediate publication on acceptance

- Inclusion in PubMed, CAS, Scopus and Google Scholar

- Research which is freely available for redistribution 